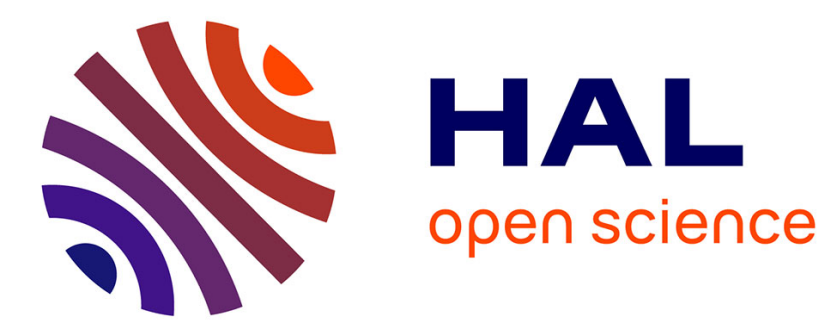

\title{
Self-organizing Multirobot Exploration through Counter-Ant Algorithm
}

Ilhem Kallel, Abdelhak Chatty, M.Alimi Adel

\section{To cite this version:}

Ilhem Kallel, Abdelhak Chatty, M.Alimi Adel. Self-organizing Multirobot Exploration through Counter-Ant Algorithm. Proceedings of the 3rd International Workshop on Self-Organizing Systems (IWSOS), Dec 2008, Austria. pp.133-144. hal-00955875

\section{HAL Id: hal-00955875 https://hal.science/hal-00955875}

Submitted on 5 Mar 2014

HAL is a multi-disciplinary open access archive for the deposit and dissemination of scientific research documents, whether they are published or not. The documents may come from teaching and research institutions in France or abroad, or from public or private research centers.
L'archive ouverte pluridisciplinaire HAL, est destinée au dépôt et à la diffusion de documents scientifiques de niveau recherche, publiés ou non, émanant des établissements d'enseignement et de recherche français ou étrangers, des laboratoires publics ou privés. 


\title{
Self-organizing Multirobot Exploration through Counter-Ant Algorithm
}

\author{
Ilhem Kallel ${ }^{1,2}$, Abdelhak Chatty ${ }^{1,2}$, and Adel M. Alimi ${ }^{1}$ \\ 1 REGIM, Research Group on Intelligent Machines, \\ National School of Engineers, \\ University of Sfax, BP W 3038, Tunisia, \\ 2 High Institute of Computer Science and Management, \\ University of Kairouan, Tunisia. \\ \{ilhem.kallel, abdelhak_chatty, adel.alimi\}@ieee.org
}

\begin{abstract}
This paper presents an evolving method for a self-organizing multirobot exploration of an unknown environment. In such problem, a big consideration is given to the coordination behavior of robots in order to achieve the common tasks in an optimal way. Ant algorithms are proved to be very useful in solving such distributed control problems. We present here a modified version of the known ant algorithm, called Counter-Ant Algorithm (CAA). Indeed, the robots'collective behavior is based on repulsion instead of attraction to pheromone, which is a chemical matter open to evaporation and representing the core of ants' cooperation. A series of experimentations with MINDSTORMS LEGO robots, and simulations under Madkit platform, in laboratory conditions similar to real ones, show the usefulness of our algorithm for self-organizing and cooperative exploration.
\end{abstract}

Key words: Counter-Ant algorithm, self-organizing multirobot, cooperative exploration, pheromone, stagnation recovery, Lego robots, Madkit.

\section{Introduction}

Multirobot systems affects our society in a fundamental way; to promote development in this field, several approaches in robotics'world have emerged and they focus on different aspects such as models of cooperation/collaboration, detection, location and exploration. However, these methods suffer from lack of robustness related to coordination between robots, resulting negative impacts on the convergence time of the operating multirobot system. To overcome these challenges, the attention of researchers has been focused on the wild world which unveiled a self-organization even more complex and harder which proves the rate of intelligence among other species. The operating results led to a new field called swarm intelligence, covering ants' algorithms. These algorithms provide powerful methods for the design of algorithms and optimization of distributed problems involving a collaborative swarm behavior [2] [4] [5] [19]. The intrusion of these algorithms in the world of robotic improve the communication quality between robots but the convergence time remains problematic [1] [6] [7] [11] [12] [13] [18] 
[20][21]. Can we go by ants algorithms in order to achieve a more efficient multirobot collaboration, while minimizing the system convergence time and realizing a best trade-off between optimization and goal to reach? We present in this paper a modified version of the known ant algorithm, called Counter-Ant Algorithm (CAA). Indeed, the robots' collaborative behavior is based on repulsion instead of attraction to pheromone, which is a chemical matter open to evaporation and representing the core of ants' cooperation. The robots' reaction consists henceforth in avoiding paths more covered by this chemical substance. In order to test the performance of our CAA, we had to look firstly for a suitable assembly of Mindstorms Lego robots to be cooperative explorers and pickers [14] [15] and an appropriate representation of pheromone [16]; secondly, for a suitable simulation platform that can represent both swarm behavior and cooperative robot behavior [3] [8] [10].

The paper is organized as follows: section 2 overviews some collaborative swarm robots based on ant algorithms. Sections 3 and 4 present our proposal for a CAA in multirobot collaboration while providing solution to stagnation situation. Section 5 and 6 present and discusse some experimentation and similation results. Finally, last section concludes the paper.

\section{Cooperative Swarm Robots based on Ant-Algorithm}

Among swarm intelligence techniques, some are mature. In fact, the observation of ants' colony led to ants algorithms [5] [6]. These revolve around a key concept called stigmergy which is an indirect activities' coordination in an unknown environment: Ants are attracted to each other thanks to chemical material called pheromone. It transpires that in an unknown environment, one of the most important problem related to multirobot systems, is to decide how to coordinate actions in order to achieve tasks in an optimal way [11]. Also, it is very important to know what needs to be accomplished and what is the number of robots required for the task. Also, among the multirobot systems, we note the emergence of self-organizing cooperative behavior. To give solution to self-organization, researchers resort to stigmergy. For example, when a robot concludes that it can not conclude a mission, it marks the unfinished task with a quantity of pheromone. More difficult the task is, the greater the amount of pheromone increases. Thus, robots will be attracted to try to accomplish this task [20]. It is clear that box pushing is more natural if it is accomplished with ants' multirobot system: In fact, the ant tries to move the food alone. it spent moments to test the food resistance by varying the orientation of its body. Changing the direction of the applied strength can be enough to really move the cargo. Should the realignment would not be sufficient, the ant frees prey and find another position to seize the cargo. If multiple replacement attempts are not successful, the ant recruits other ants [11]. Many works such as cited by Kube and Zhang [12][13] are based on ant algorithm to solve transportation problem. Other research consists to change the ant algorithm using a new technique for multirobot pheromone placement. This technique enables robots to place more pheromones in the tasks 
that are about to be fulfilled: A very large amount of pheromone is placed in the balls that are almost being moved or are nearly to their destinations, while a small amount of pheromone is placed in the balls, which require a great force to make them move. This helps to attract other robots to complete the task [20]. Except that the amount of pheromone is constant, whereas in [1] [21] is variable and depends on task difficulty and the robots strength. The problem of task allocation in the field of cooperative robotics was led by several studies especially in unknown environment. Each robot has to adapt to its environment without any training stage. Accordingly, swarm intelligence allows self-organization into an unfamiliar environment and adapting behaviors through simple individuals' interactions.

\section{Multirobot Exploration based on Counter-Ant behavior}

We present here another view of swarm intelligence inspired from ant colony optimization; it is based on a modified version of ant algorithm called CounterAnt-Algorithm (CAA). Figure 1 expresses the pseudo-code of our CAA: the modules task_Achievement encapsulates the objective operation (i.e; cleaning, collecting, picking, hunting, etc.) pheromone_Update formulates the evaporation phenomenon of the pheromone and daemon_Counter_Reactions expresses the reaction of the robots, which is repulsion instead of attraction to pheromone; it includes also the solution of stagnation recovery. To feign well our algorithm, we propose a nominal scenario describing the behavior of self-organized ant-robots explorers (see figure 2).

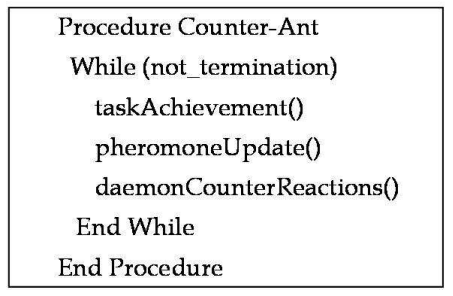

Fig. 1. Counter-Ant behavior pseudo code

Given the evaporation property of the pheromone, the paths eventually disappear which enables ants expand their scanning areas. In other words, as old paths marked by the pheromone no longer exist, then the ants can reach, when they move randomly, new places, which were not detected by other ants.

\subsection{Pheromone Update}

Ants move and marking paths with a constant amount of pheromone, after a fixed time, the paths of pheromone begin to disappear. The amount of pheromone in each zone of the cleaning environment is updated according the equation 1. 


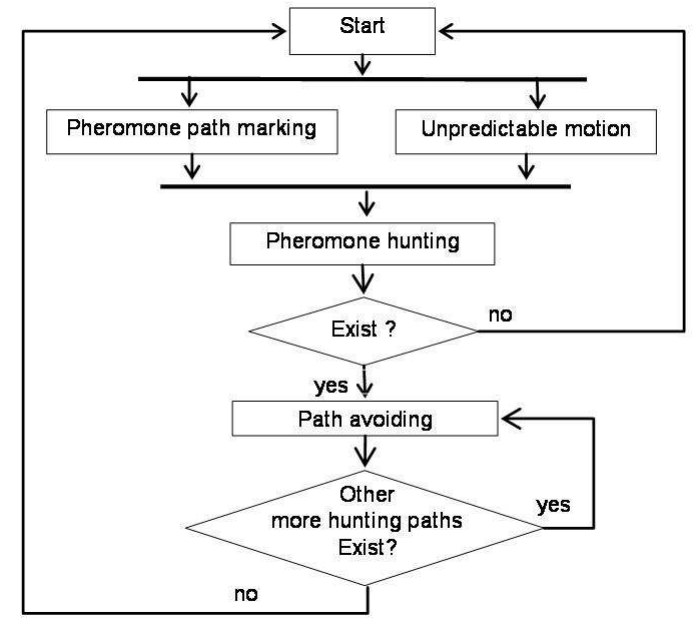

Fig. 2. Collaborative exploration behavior based on CAA

$$
\tau_{x, y}=\rho \tau_{x, y}+\Delta \tau_{x, y}
$$

where, $\tau$ is the amount of pheromone in the zone of coordinates $x, y ; \rho$ is the rate of pheromone evaporation and $\delta \tau$ is the amount of pheromone deposited when an ant go through the zone $x, y$.

\section{Resolution of Stagnation with the Pheromone}

A problematic case appears: the stagnation of robots motion due to dead ends. This means that robots lost totally the possibility of moving in their unknown environment. We propose to include in our CAA a stagnation recovery which allows the robots to overtake dead ends and to resolve stagnation problems. The unpredictable motion of ants' robots is accompanied by a secretion of pheromone so that the other ants do not borrow the already marked paths. After a while, the ant-hill (the robot environment) will be blocked by pheromone, this can create what we call stagnation situations (SS). In our system, the SS are similar to dead ends [1][11][20]: they refer to situations where ants are surrounded by the pheromone; this causes the blocking of their actions because they have to avoid the perceived pheromone.

\subsection{Stagnation Recovery using Evaporative Pheromone}

The preservation of the pheromone evaporation property has allowed the ants to enlarge their sweeping areas one hand, and on the other hand, reduced the stagnation situations since the paths end up disappearing, which allows the ants to continue exploring the dynamic environment. Evaporation of the pheromone 
is not instantaneous; it requires some time to be completed. This helps to inform, in an interval of time, the maximum number of ants already passed. However, the lifetime of the pheromone is itself a factor of stagnation situations: ants can be found surrounded by tracks of pheromones that have not yet evaporated. The first idea for resolving these problematic situations is to take advantage from evaporation: ants stuck and have to wait till evaporation of one condemning issue, but this causes a lack of exploring workforce! If all ants are imprisoned, our algorithm will enter a waiting phase until their release. This issue will negatively affect the convergence time of our system because it will be affected by pending active ants that are in stagnation situations.

\subsection{Stagnation Recovery using Positions' Pheromone}

The solution is then to give ant the ability to become localized according to pheromone position: if its location is parallel or confused to the pheromone, it has to change direction in order to find another way, elsewhere it may be in the presence of other ants. On the other hand, if its location is not parallel, it can move to overcome this stagnation. In this case, the ant doesn't avoid the pheromone since both don't have the same direction and therefore we no longer refer to congestion areas. This idea is encapsulated in daemonCounterReactions() function

\section{Experimentation results with Lego Robots}

The inter-robot cooperation takes place in a totally unknown environment. It consists of chemical-sensitive navigating platform containing some objects to pick up and two Lego Mindstorms ant-robots. In order to test the performance of our CAA, we had to look for a suitable assembly of Lego Mindstorms robots 3 [14] [15] to be cooperative explorers and cleaners [3][8] and an appropriate materialization of pheromone [16]. We utilize magic ink (see figure 3), which is a chemical substance made up of ethanol, thymolphthalein, demineralized water and sodium hydroxide capable of producing chemical traces of disappearing blue until their total fading.

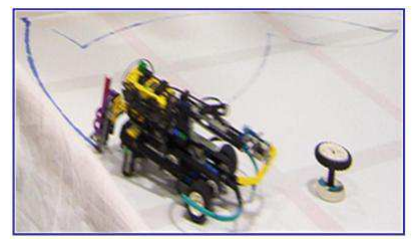

Fig. 3. Path traces and their evaporation

\footnotetext{
${ }^{3}$ http://mindstorms.lego.com/eng/products/ris/index.asp
} 
We construct our ant-robots from Lego Mindstorms of Robotics Invention System series. One box contains one RCX 2.0 (Robotics Command System) which presents the intelligent programmable part. Using these boxes, we were able to build a twin Lego robots. In addition to the various Lego parts, each one is built by one RCX box, carpet of loading and unloading objects, light, contact sensors and the magical ink. This construction may well realize the basic functions of our algorithm which can be summarized in the motion, loading/unloading objects, and the detection of pheromone, obstacles and borders. Figure 4 presents some experimental scenarios photographed in the occasion of Sfax University fair (Tunisia).

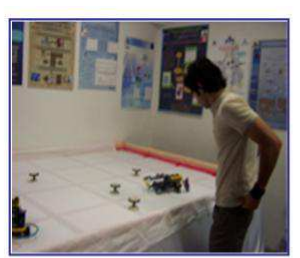

(a)

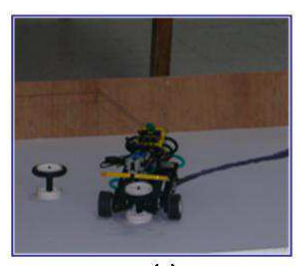

(c)

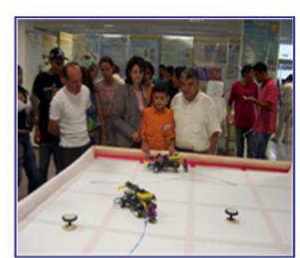

(b)

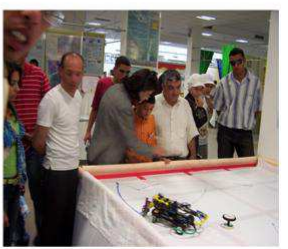

(d)

Fig. 4. Some experimental scenarios (a) Robots starting, (b) Motion with ink trace, (c) Object cargo, and (d) Evaporation of some paths

A series of experimentations show the usefulness of our algorithm for selforganizing and cooperative exploration and cleanup. We tried to clean the experimental environment with only one robot and then with two robots. We noticed, in all cases, a temporal gain around $40 \%$ and a spectacular self partitioning of the explored area. In order to analyse the behaviour of robots during the execution of the CAA, it is judicious to highlight the individual behaviour of a robot. The robot moves randomly when running the treadmill. It should be mentioned that the robots are blind due to the absence of a mechanism that can detect remote object (figure 5a). Figure 5b shows the object cargo found on the robot path. This operation is provided via the treadmill. Figure $5 \mathrm{c}$ and $5 \mathrm{~d}$ show that the treadmill charges object till the touch sensor. The robot carries the object outside, stops, removes the object and performs a reverse to change direction (figure 5e and 5f). It continues moving randomly for cleaning, There are two cases that arise showing the reaction of the robot according to the magic-ink (pheromone): if the trace is perpendicular to the robot path (figure $5 \mathrm{~g}$ ), the 
robot continues crossing (figure 5h). But if the trace coincides with the robot trajectory (figure 5i), the robot moves away (figure $5 \mathrm{j}$ ). The robot is equipped with a front bumper, which allows it to detect static and moving obstacles such as another robot (figure 5k); in this case, both have to change direction (figure 5l). The conservation of the property of evaporation generates disappearance of the pheromone traces, which makes it possible the robots to increase their sweeping zones.

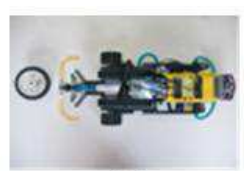

(a)

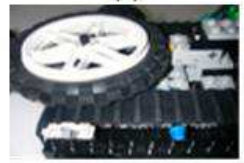

(d)

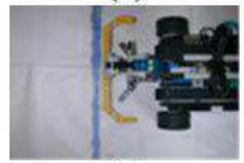

(g)

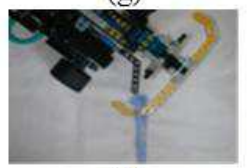

(j)

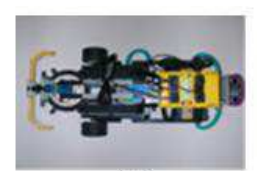

(b)

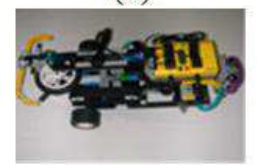

(e)

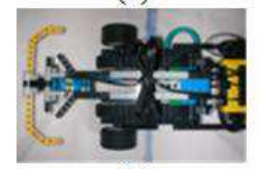

(h)

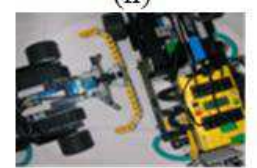

(k)

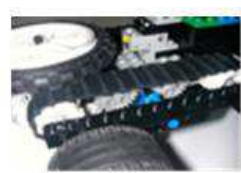

(c)

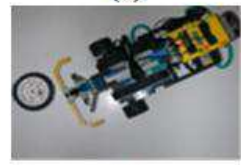

(f)

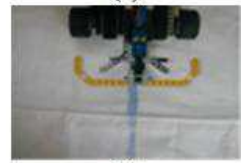

(i)

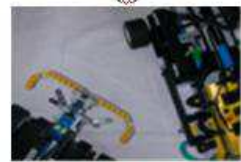

(1)

Fig. 5. Individual behavior of our ant-robot

The experimental phase has shown an acceptable assembly of reactive and cooperative cleaning robots and their adaptability to functions requested in our counter-Ant algorithm: both Lego Mindstorms robots are designed to move randomly for objects hunting, marking paths by an evaporating Magic ink. To identify ink plots, robots use light sensors having however some failures due to the non-precision values that depend on the ambient light in the room. A series of experimentations allowed us to improve our program. Indeed, we have been able to improve speed motion and load; this precludes our construction holds some constraints related to the adaptability degree of objects to the treadmill.

\section{Simulation results under Madkit platform}

We carried our interest in the cleaning field and we noticed that cleaning an unknown environment by a multirobot system, apparently simple, conceals some 
complexity in terms of inter-robots coordination and efficiency. In fact, we noticed a congestion in the cleaning zones which influences negatively the convergence time. To better discuss this function, we implemented our CAA in the simulation platform Madkit ${ }^{4}$. Collaboration between robots is done in a completely unknown environment. It is made up mainly by objects and cleaning robots which are implemented and generated by the simulation platform. These cleaning robots are provided by a skeletal sensor enabling them to detect the pheromone trace. The robots are completely blind and their motion is completely random. For the cleaning operation, it is question to remove meted. This enables us to focus on our Counter-Ants Algorithm to emphasize our multirobot collaboration method. Moreover, we can consider also that our robots are provided by tanks able to contain a collection of garbage. Figure 6 a presents the simulation environment at the starting time $\mathrm{t}=0$ containing five cleaning robots and 80 objects. The time of pheromone evaporation is firstly fixed to $45 \mathrm{~s}$. Figure $6 \mathrm{~b}$ is a screen caption after a running time $\mathrm{t}=18,63 \mathrm{~s}$; according to pheromone traces, we can see that the robots move away or cross other marked paths, since the robots behavior is based on repulsion instead of attraction to pheromone. The cleaning robot motion is accompanied by the activation of pheromone detectors in order to locate the recent robots trajectories in the environment. The figure $6 \mathrm{c}$ shows the end of the cleaning operation after $\mathrm{t}=135,20 \mathrm{~s}$. As the paths end up disappearing, it is what explains the existing number of pheromone traces in the environment, and after an additional 45s, all first pheromone traces will disappear (see Figure 6d).

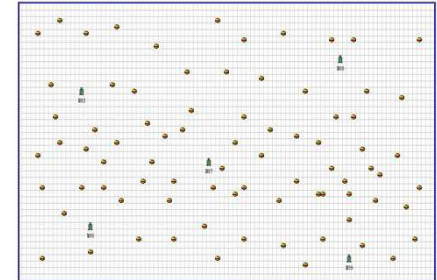

(a)

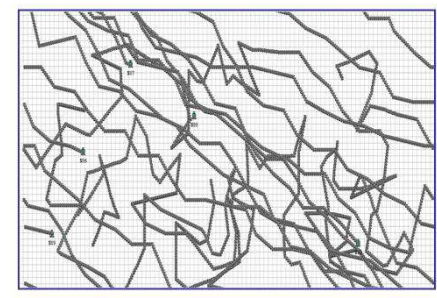

(c)

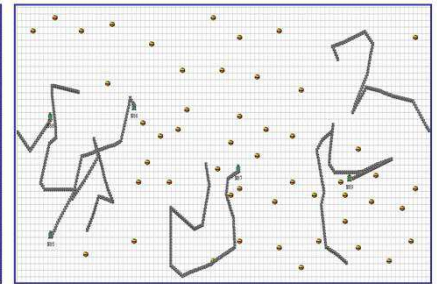

(b)

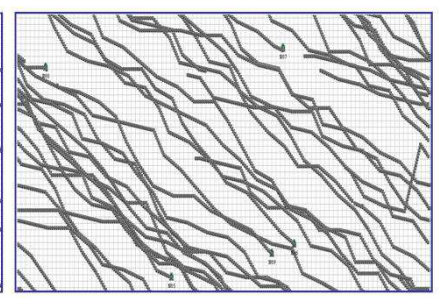

(d)

Fig. 6. Some simulation screen captions

\footnotetext{
$\overline{{ }^{4} \text { http://www.madkit.org }}$
} 


\subsection{Influence of Parameters' Variation}

Figure 7 shows (from 1 to 2 robots) that in spite of the fixed time of evaporation to $8 \mathrm{~s}$, the convergence time is improved, from $1816,09 \mathrm{~s}$ with only one robot, to $1426,17 \mathrm{~s}$ with two robots. This emphasizes the effectiveness of swarm robotics in the realization of cleaning operation. This figure also shows (from 2 to 5 robots) that the long life of pheromone paths guarantees the reduction of obstruction zones, since their presence allow robots to recognize previously explored zones. This is ensured thanks to the reaction of robots with respect to the pheromone which consists of repulsion and not attraction. The increase in the number of robots with the persistence of the traces does nothing but improve time of convergence since the robots any more will not seek objects in open zones but rather in closed spaces.
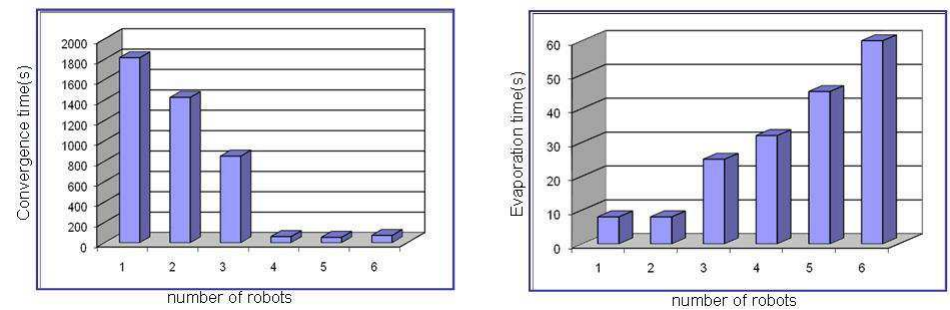

Fig. 7. Evaluation of the convergence time following the variation of the number of robots and the evaporation time.

In terms of stagnation recovery, we observe that in spite of the long life of the pheromone evaporation, the robots are able to progress while accelerating the convergence time. However, (from 5 to 6 robots) the persistence of the pheromone trace for a long life (45s to $60 \mathrm{~s}$ ) increase the convergence time. In fact, the environment will be encumbered by pheromone traces, thing that limits the robot motion and causes slowness of convergence time.

\subsection{Exploration Behavior vs. Random Behavior}

To better point out the effectiveness of counter-ant behavior and stagnation recovery, we propose to compare it with a random behavior. Both simulation environments contain 80 objects and 5 robots. $45 \mathrm{~s}$ is the time of pheromone evaporation for counter-ant behavior. The figure 4a shows the initial state of the random system. The figures $8 \mathrm{~b}$ and $4 \mathrm{c}$ show the random operation of cleaning where lines represent robotsmotion traces and cluttering lines are due to pure rondom motion. The figure $8 \mathrm{~d}$ shows a screen caption after $\mathrm{t}=843,56 \mathrm{~s}$. Whereas, the conversion time with our CAA is $t=135,20$ s according to the figure $6 \mathrm{~d}$. 


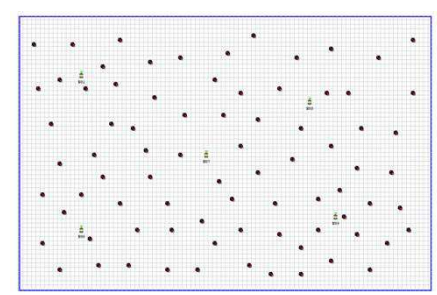

(a) $\mathrm{t}=0 \mathrm{~s}$

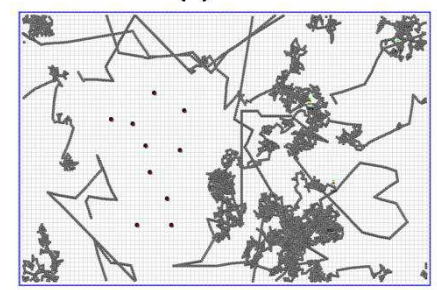

(c) $t=523,25 \mathrm{~s}$

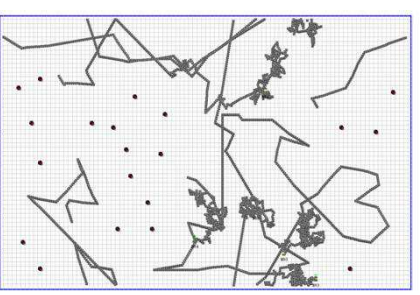

(b) $t=135,72 \mathrm{~s}$

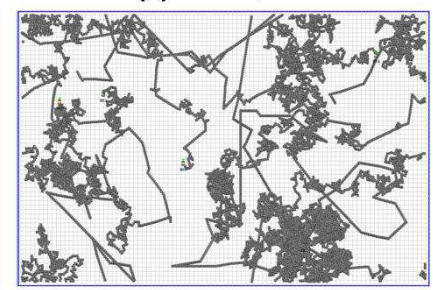

(d) $t=843,56 \mathrm{~s}$

Fig. 8. Simulation of hazardous behaviors

\subsection{Counter-Ants vs. Alliance}

The Alliance architecture suggested by Parker [17] based on faults tolerance is a supervised architecture which consists on collaborating robots to clean two sources of rubbish. The communication between robots is done through the diffusion of messages about sources localization via a supervision system. Alliance is certainly based on the faults tolerance however, the supervision of the robots present in itself a limit of the solution. It is important to mention that the quality of the exploration of the environment was not approached. Figure 9 summarizes this assessment

\begin{tabular}{llllllll}
\hline Approaches & No $\begin{array}{l}\text { Stigmergy: } \\
\text { pheromone }\end{array}$ & Yes pheromone & assured & Yes & $\begin{array}{l}\text { Exploration } \\
\text { and } \\
\text { cleanup }\end{array}$ & Medium \\
$\begin{array}{l}\text { Counter- } \\
\text { Ants }\end{array}$ & $\begin{array}{l}\text { sensorst } \\
\text { diffusion } \\
\text { of } \\
\text { Messages }\end{array}$ & Yes Bliance & Yehavior & - & No & Cleanup & Long \\
\hline
\end{tabular}

Fig. 9. Counter-Ants vs. Alliance 
Alliance presents a specific solution for rubbish cleaning gathered and not scattered. However, our CAA can be well applied to scattered objects cleaning in unknown environment and to any exploration problem. It is an unsupervised solution with stigmergy as the base of communication and collaboration. In fact, it possible to solve the exploration problem and the dilemma convergence time/goal to reach.

\section{Concluding Remarks}

This work consists to present and apply a modified version of the known ant algorithm, called Counter-Ant Algorithm (CAA). It consists on a new collaborative behaviour in relation to the pheromone in a multirobot system. This technique enables robots to place the pheromone while moving randomly. This will no longer attract other robots, but rather to avoid motion paths (already explored and cleaned areas) by other robots. As a result, this method allows improving system efficiency and minimizes the creation of congested areas. As the old paths marked by the pheromone will disappear, new places become open to explore, which were not detected by other ant-robots. This leads us to say that the subdivision of the area to explore is self organized [9]. We present also a solution for the stagnation recovery which allows the robots to overtake dead ends. We simulate, implemented and experiment our algorithm in laboratory conditions similar to real ones. A series of simulations and experimentations show the usefulness of our algorithm for adaptive and cooperative exploration (including cleaning, picking, etc). As perspectives, we propose firstly to look for a more natural and less glaring representation of pheromone, Secondly, we intend to hybridize our algorithm with a soft computing technique, such as fuzzy rule base system, in order to surround more fluently the stagnation situations.

\section{Acknowledgements}

The authors thank the Tunisian General Direction of Scientific Research and Technological Renovation (DGRSRT), under the ARUB program 01/UR/11-02, Tunisia.

\section{References}

1. Borzello, E., Merkle, L.D.: Multi-Robot Cooperation Using the Ant Algorithm with Variable Pheromone Placement, Proceedings of the IEEE, 2005

2. Casillas, J., Cordn, O. , Fernndez de Viana, I. , Herrera, F. : Learning cooperative linguistic fuzzy rules using the best-worst ant system algorithm, International Journal of Intelligent Systems 20:4, 2005.

3. Cao, Y.U., Fukunaga, A.S., Khang, A.B. : Cooperative mobile robotics: antecedents and directions, Autonomous Robots 4 (1) (1997) 7-27.

4. Dorigo, M., DiCaro, G. : The Ant Colony Optimization Meta-Heuristic, New Ideas in Optimization 1999. (New York: McGraw-Hill) 
5. Dorigo, M., Stutzle, T. : Ant colony optimization. Cambridge, MA, USA: The MIT Press, 2004.

6. Dudek, G., Jenkin, M. , Milios, E. , Wilkes, D. : A taxonomy for swarm robots, Intelligent Robots and Systems, IROS '93. Proc. of the 1993 IEEE/RSJ,Vol. 1, July 1993, pp. 441-447.

7. Holland, O. , Melhuish, C.: Stigmergy, self-organization, and sorting in collective robotics, Artificial Life, vol. 5, no. 2, 1999, pp. 173-202.

8. Johnson, J. , Sugisaka, M. : Complexity science for the design of swarm robot control systems, Industrial Electronics Society, 2000. IECON 2000. 26th Annual Conference of the IEEE, Vol.1, Oct. 2000, pp. 695-700

9. Kallel, I., Mezghani, S. , Alimi, A.M. : Towards a Fuzzy Evaluation of the Adaptivity Degree of an Evolving Agent, Proc. of the 3rd International Workshop IEEE GEFS, March 2008, pp. 29-34.

10. Kasabov, N., Filev, D. : Evolving intelligent systems: methods, learning, applications, in Proc. Int. Symposium on Evolving Fuzzy Systems EFS, 2006, pp. 8-18.

11. Kube, C.R., Bonabeau, E. : Cooperative transport by ants and robots, Robotics and Autonomous Systems, vol. 30, 2000, pp. 85-101.

12. Kube, C.R., Zhang, H. : Collective robotics: From social insects to robots, Adaptive Behavior 2, 189-218, 1994

13. Kube, C.R, Zhang, H. : Stagnation recovery behaviors for collective robotics, in: Proceedings of the 1994 IEEE/RSJ/GI International Conference on Intelligent Robots and Systems, IEEE Computer Society Press, Los Alamitos, CA, 1995, pp. 1883-1890.

14. Lund, H.H. : Adaptive robotics in entertainment, Applied Soft Computing, 2001.

15. Lund, H.H., Pagliarini, L. : RoboCup with LEGO MINDSTORMS, in: Proceedings of the International Conference on Robotics and Automation (ICRA 2000), IEEE Press, NJ, 2000.

16. Mamei, M., Zambonelli, F. : Pervasive Pheromone-Based Interaction with RFID Tags, ACM Transactions on Autonomous and Adaptive Systems, 2(2):1-28, 2007.

17. Parker, L. : ALLIANCE: An Architecture for Fault Tolerant Multi-Robot Cooperation, IEEE Transactions on Robotics and Automation, vol. 14, no. 2, 1998, pp.220-240.

18. Purnamadjaja, A.H. , Russell, R.A. : Pheromone communication: implementation of necrophoric bee behaviour in a robot swarm, Robotics, Automation and Mechatronics, 2004 IEEE, Dec. 2004, Vol. 2, pp.638-643.

19. Szu, H., Chanyagorn, P., Hwang, W., Paulin, M., Yamakawa, T. : Collective and distributive swarm intelligence: evolutional biological survey, International Congress Series, Volume 1269, August 2004, Pages 46-49.

20. Yingying, D., Yan, H., Jingping, J. : Multi-Robot Cooperation Method Based On The Ant Algorithm, Proc. of the IEEE, 2003.

21. Zhang, D., Xie, G., Yu, J.i, Wang, L. : Adaptive task assignment for multiple mobile robots via swarm intelligence approach, Robotics and Autonomous Systems, vol. 55, no. 7, 2007, pp. 572-588. 